\title{
Exposing points on the boundary of a strictly pseudoconvex or a locally convexifiable domain of finite 1-type
}

\author{
K. Diederich, J. E. Fornæss and E. F. Wold*
}

September 27, 2018

\begin{abstract}
We show that for any bounded domain $\Omega \subset \mathbb{C}^{n}$ of 1-type $2 k$ which is locally convexifiable at $p \in b \Omega$, having a Stein neighborhood basis, there is a biholomorphic map $f: \bar{\Omega} \rightarrow \mathbb{C}^{n}$ such that $f(p)$ is a global extreme point of type $2 k$ for $f(\bar{\Omega})$.
\end{abstract}

\section{Introduction}

In this paper we consider bounded locally convexifiable domains $\Omega$ in $\mathbb{C}^{n}$ of finite 1-type whose closures $\bar{\Omega}$ admit a Stein neighborhood basis. Here the term "locally convexifiable near $p \in b \Omega$ " means that there are a neighborhood $V$ of $p$ and a one-to-one holomorphic map $\Phi: V \rightarrow \mathbb{C}^{n}$ such that $\Phi(\Omega \cap V)$ is convex. For the notion of finite type we refer to [2]. Strongly pseudoconvex domais are examples of such domains. We will first prove the following:

Theorem 1.1 Let $\Omega \subset \mathbb{C}^{n}$ be a bounded domain which is locally convexifiable and has finite type $2 k$ near a point $p \in b \Omega$. Assume further that $b \Omega$ is $\mathcal{C}^{\infty}$. smooth near $p$, and that $\bar{\Omega}$ has a Stein neighborhood basis. Then there exists a holomorphic embedding $f: \bar{\Omega} \rightarrow \overline{\mathbb{B}}_{k}^{n}$, where $\mathbb{B}_{k}^{n}=\left\{z \in \mathbb{C}^{n}:\left|z_{n}\right|^{2}+\left\|z^{\prime}\right\|^{2 k}<1\right\}$, such that

1. $f(p)=e_{n}=(0, \ldots, 0,1)$, and

2. $\left\{z \in \bar{\Omega}: f(z) \in b \mathbb{B}_{k}^{n}\right\}=\{p\}$.

If $k=1$, i.e., if $b \Omega$ is strongly pseudoconvex near $p$, it is enough to assume that $b \Omega$ is $\mathcal{C}^{2}$-smooth near $p$.

Definition 1.2 Let $\Omega \subset \mathbb{C}^{n}$ be a domain and let $p \in b \Omega$ be a point. We say that $p$ is a globally exposed $2 k$-convex point if there exists an affine linear map $f$ as in the previous theorem.

\footnotetext{
${ }^{*}$ Supported by the NFR grant 209751/F20
} 
One of our motivations for proving this theorem is the special case of strictly pseudoconvex domains. In this case the theorem answers a question posed by Fusheng Deng (private communication), and it is a step to study squeezing functions on bounded strongly pseudoconvex domains (see [3]).

Another motivation consists in the construction of a $\mathcal{C}^{\infty}$ family of local holomorphic support functions $\hat{S}(z, \zeta) \in \mathcal{C}^{\infty}\left(\mathbb{C}^{n} \times \partial D\right)$ for locally convexifiable domains of finite type $2 k$ with Stein neighborhood basis as explained in [4]. It has been asked several times whether these support functions can always be chosen such that they are globally supporting for the given domains.

However, it has to be asked in which way precisely this question should be answered. As far as we can see, there are at least the following different possibilities, each of them leading to quite different answers:

1. Our original support surfaces are defined only locally. The danger might be, for instance, that after a while they fall back into the inside of the domain or, at least, become tangent at certain points, that are further away. However, this danger can be avoided by applying a simple standard $\bar{\partial}$-argument to the defining functions of the support functions. Then we get new support functions which are well-defined in a possibly narrow Stein neighborhood of $\bar{\Omega}$.

2. Asking for more might mean that we really want globally defined support surfaces, i.e., support surfaces which are closed smooth complex hypersurfaces in $\mathbb{C}^{n}$, touching $b \Omega$ only from the outside at one distinguished boundary point. It is clear that this requires a much stronger hypothesis on the domain. Namely, we will assume that the given domain has a Runge neighborhood basis and is locally convexifiable of finite type near 0 . It is one of the main results of this article (Theorem 1.3) that such closed global support surfaces then always do exist. Under suitable regularity assumptions on $b \Omega$ (namely $b \Omega$ has to be $\mathcal{C}^{\infty}$-smooth) smooth $\mathcal{C}^{\infty}$-families of such supporting hypersurfaces do indeed exist (Theorem 1.4).

In this part of the work we will prove the following statement:

Theorem 1.3 Assume in addition to the hypotheses in Theorem 1.1 that $\bar{\Omega}$ has a Runge and Stein neighborhood. Then the map $f$ can be chosen as a global automorphism of $\mathbb{C}^{n}$. A special case of this are convex domains of finite 1-type.

Finally, in the case of bounded and smooth convex domains, we prove a version of Theorem 1.1 with parameters:

Theorem 1.4 Let $\Omega \subset \mathbb{C}^{n}$ be a smooth and bounded convex domain of finite type $2 k$. There exists a smooth family $\psi_{\zeta} \in A u t_{h o l} \mathbb{C}^{n}, \zeta \in b \Omega$, such that $\psi_{\zeta}(\zeta)$ is a globally exposed $2 k$-convex boundary point for the domain $\psi_{\zeta}(\Omega)$.

The structure of the article is as follows: In Section 2 we recall some local properties of convexifiable domains due to the two first authors. In Section 3 we prove Theorem 1.1. In Section 4 we prove Andersén-Lempert theorems with 
parameters needed to prove Theorem 1.4, which we will do in Section 5. Finally, in Section 6, we give a brief sketch of how to prove Theorem 1.3 based on the arguments in Sections 3 and 5.

\section{Local properties of convexifiable domains}

Let $\Omega$ be a bounded $\mathcal{C}^{\infty}$-smooth domain in $\mathbb{C}^{n}$. In this section we recall the main facts about supporting hypersurfaces constructed in [4] For this we suppose that there is an open set $V \subset \mathbb{C}^{n}$ such that $b \Omega \cap V$ is convex. Near any point $\zeta_{0} \in b \Omega \cap V$ there is an open neighborhood $V_{\zeta_{0}}$ of $\zeta_{0}$, and a choice of a $\mathcal{C}^{\infty}$-family of coordinate changes $\left\{l_{\zeta}(z): \zeta \in b \Omega \cap V_{\zeta_{0}}\right\}$ composed of a translation and a unitary transformation, such that, for each $\zeta \in b \Omega \cap V_{\zeta_{0}}, l_{\zeta}(\zeta)=0$ and the unit outward normal vector $n_{\zeta}$ at $\zeta$ is turned by $l_{\zeta}$ into the unit vector $(1,0, \ldots, 0)$. In particular, $T_{\zeta}^{\mathbb{C}} b \Omega$ becomes in the new coordinates $\tilde{z}=l_{\zeta}(z)$ associated to $\zeta$ just $\left\{\tilde{z}_{1}=0\right\}$. The following is proved in [4]:

Theorem 2.1 In the situation just described, assume that $b \Omega \cap V$ is of finite 1-type $2 k$, and let $\tilde{V} \subset \subset V$. Then there exists a function $\widehat{S}(\zeta, z) \in \mathcal{C}^{\infty}((b \Omega \cap$ $\left.\tilde{V}) \times \mathbb{C}^{n}\right)$, and constants $r, c>0$, such that the following holds: for any choice of coordinate changes $l_{\zeta}$ as above, the function $S(\zeta, z):=\widehat{S}\left(\zeta, l_{\zeta}^{-1}(z)\right)$ is equal to

$$
S_{\zeta}(z)=3 z_{1}+K z_{1}^{2}+g_{\zeta}\left(z^{\prime}\right) \text {, where }\left(z_{1}, z^{\prime}\right) \text { are coordinates on } \mathbb{C}^{n},
$$

and satisfies the estimate

$$
\operatorname{Re} S_{\zeta}(z) \leq-c\left(\left|z_{1}\right|^{2}+\left\|z^{\prime}\right\|^{2 k}\right)
$$

for all $z \in B_{r} \cap l_{\zeta}(\Omega)$.

Note that if the domain $\Omega$ is convex, we get a $\mathcal{C}^{\infty}$-smooth function $\widehat{S}(\zeta, z)$ on $b \Omega \times \mathbb{C}^{n}$.

\section{The proof of Theorem 1.1}

The proof of Theorem 1.1 is reduced to the two Lemmas in this section, Lemma 3.1 and Lemma 3.3 .

We let $e_{1}, \ldots, e_{n}$ be the standard basis for the complex vector space $\mathbb{C}^{n}$ and put $f_{j}:=i \cdot e_{j}$ so that $e_{1}, f_{1}, \ldots, e_{n}, f_{n}$ is a real basis. We denote the coordinates on $\mathbb{C}^{n}$ by $z_{j}=x_{2 j-1}+i x_{2 j}$, we let $\mathbb{C}_{n}$ denote the complex line $\mathbb{C}_{n}=\left\{z \in \mathbb{C}^{n}\right.$ : $\left.z_{1}=\ldots=z_{n-1}=0\right\}$ and we let $\pi_{n}$ be the orthogonal projection to $\mathbb{C}_{n}$.

Our proof uses a technique from [8] invented for exposing points on a bordered Riemann surface in order to produce a proper holomorphic embedding (see also [6] Sections 8.8 and 8.9). We suppose that $\Omega$ is convexifiable near some point $p$ on its boundary. Then we get the following situation: 
Lemma 3.1 For any $p \in b \Omega$ there exists $\Phi \in$ Aut $_{h o l} \mathbb{C}^{n}$ such that the following hold

1. $\Phi(p)=0$ and $T_{0}(b \Phi(\Omega))=\left\{x_{2 n}=0\right\}$

2. The outward normal to $b \Phi(\Omega)$ at the origin is $f_{n}$,

3. Near the origin we have that $b \Phi(\Omega)$ is $k$-convex at the origin in the following sense: The domain $\overline{\Phi(\Omega)} \subset\left\{z \in \mathbb{C}^{n}: x_{2 n}-f\left(z^{\prime}, x_{2 n-1}\right) \leq 0\right\}$ with $f\left(z^{\prime}, x_{2 n-1}\right) \geq c\left(\left\|z^{\prime}\right\|^{2 k}+x_{2 n-1}^{2}\right), c>0$ and

4. $\overline{\Phi(\Omega)} \cap\left\{z \in \mathbb{C}^{n}: z_{1}=\ldots=z_{n-1}=x_{2 n-1}=0, x_{2 n} \geq 0\right\}=\{0\}$

Definition 3.2 When condition (3) is satisfied near the origin we will refer to the origin as a strictly $2 k$-convex boundary point.

Proof: It follows by Corollary 2.4 in $[4$ that there exists an open neighborhood $U_{p}$ of $p$ and an injective holomorphic map $\psi: U_{p} \rightarrow \mathbb{C}^{n}$ such that $\psi\left(\Omega \cap U_{p}\right)$ satisfies (1)-(3). Choosing an appropriate neighborhood $V_{p} \subset U_{p}$ of $p$, it follows that $\psi$ is approximable by automorphisms $\phi$ of $\mathbb{C}^{n}$ uniformly on $V_{p}$ (see Section 4 ) and that $\psi(\Omega)$ is strictly k-convex near $\Phi(p)$ if $\phi$ is close enough to $\psi$.

We proceed to achieve (4). Let

$$
\Gamma:=\left\{z \in \mathbb{C}^{n}: z_{1}=\ldots=z_{n-1}=x_{2 n-1}=0, x_{2 n} \geq 0\right\},
$$

and let

$$
\Gamma_{0}:=\left\{z \in \mathbb{C}^{n}: z_{1}=\ldots=z_{n-1}=x_{2 n-1}=0,0 \leq x_{2 n} \leq 1\right\} .
$$

Choose an $R>0$ such that $\bar{\Omega} \subset \mathbb{B}_{R}^{n}$. By $\left[7\right.$ there exists $\psi_{1} \in$ Aut $_{\text {hol }}\left(\mathbb{C}^{n}\right)$ such that $\psi_{1}(z)=z+O\left(\|z\|^{2 k+1}\right)$ as $z \rightarrow 0$, such that $\psi_{1}\left(\Gamma_{0}\right) \cap \bar{\Omega}=\{0\}$, and such that $\psi_{1}(q) \in \mathbb{C}^{n} \backslash \mathbb{B}_{R}^{n}$, where $q$ denotes the endpoint of $\Gamma_{0}$ other than the origin. Consider the set $\psi_{1}^{-1}\left(\overline{\mathbb{B}_{R}^{n}}\right) \cap \Gamma \subset \Gamma \backslash\{q\}$. Since $\psi^{-1}\left(\overline{\mathbb{B}_{R}^{n}}\right)$ is polynomially convex we have that $\left(\mathbb{C}^{n-1} \times\{i\}\right) \backslash \psi_{1}^{-1}\left(\overline{B_{R}^{n}}\right)$ is connected, and so using Weierstrass approximation theorem, we may construct a holomorphic shear map $\psi_{2}(z)=\left(z_{1}+f_{1}(z), \ldots, z_{n-1}+f_{n-1}\left(z_{n}\right), z_{n}\right)$ such that $\psi_{2}$ is close to the identity on $\Gamma_{0}$, tangent to the identity to order $2 \mathrm{k}+1$ at the origin, and therefore not destroying strict k-convexity at 0 , and such that $\psi_{2}\left(\overline{\left(\Gamma \backslash \Gamma_{0}\right)} \cap \psi_{1}^{-1}\left(\bar{B}_{R}^{n}\right)=\emptyset\right.$. So $\left(\psi_{1} \circ \psi_{2}\right)(\Gamma) \cap \bar{\Omega}=\emptyset$, and we set $\Phi=\left(\psi_{1} \circ \psi_{2}\right)^{-1}$.

Lemma 3.3 Let $W \subset \mathbb{C}^{n}$ be a bounded domain with $0 \in b W$ and assume that the following hold

(i) $\bar{W}$ has a Stein neighborhood basis,

(ii) $W$ is strictly $k$-convex near the origin,

(iii) $\bar{W} \cap \Gamma_{0}=\{0\}$ with $\Gamma_{0}$ defined as in the proof of Lemma 3.1. 
Then for any open set $\tilde{V}$ containing $\Gamma_{0}$ and any small enough open set $V \subset \tilde{V}$ containing the origin, there exist a sequence of holomorphic embeddings $f_{j}$ : $\bar{W} \rightarrow \mathbb{C}^{n}$ such that the following holds

(1) $f_{j} \rightarrow$ id uniformly on $\bar{W} \backslash V$ as $j \rightarrow \infty$

(2) $f_{j}(V) \subset \tilde{V}$ for all $j$,

(3) $f_{j}(0)=f_{n}$ for all $j$

(4) $\operatorname{Im}\left(\pi_{n}\left(f_{j}(z)\right)\right)<1$ for all $z \in(\bar{W} \cap V) \backslash\{0\}$ and

(5) $f_{j}(W)$ is strictly $k$-convex at $f_{j}(0)$.

Proof: We let $\tilde{\Omega} \subset \mathbb{C}_{n}$ denote the domain $\tilde{\Omega}:=\left\{z_{n} \in \Delta_{\varepsilon}: x_{2 n}<f\left(0, \ldots, 0, x_{2 n-1}\right\}\right)$. For some small $\delta>0$ we define the following sets: $A:=\left\{z \in \bar{W} \cap \overline{\mathbb{B}_{\varepsilon}^{n}}: x_{2 n} \geq-\delta\right.$ and $B:=\left\{z \in \bar{W} \cap \overline{\mathbb{B}_{\varepsilon}^{n}}: x_{2 n} \leq-\frac{\delta}{2}\right\} \cup \bar{W} \backslash \mathbb{B}_{\varepsilon}^{n}$. Then $\overline{A \backslash B} \cap \overline{B \backslash A}=\emptyset$ (if $\delta$ is small) and by Theorem 4.1 in [5], for any open set $\tilde{C}$ containing the set $C:=A \cap B$ there exist open sets $A^{\prime}, B^{\prime}, C^{\prime}$ with $A \subset A^{\prime}, B \subset B^{\prime}$ and $C \subset C^{\prime} \subset A^{\prime} \cap B^{\prime} \subset \tilde{C}$, such that if $\gamma: \tilde{C} \rightarrow \mathbb{C}^{n}$ is injective holomorphic, and sufficiently close to the identity, then there exist holomorphic injections $\alpha: A^{\prime} \rightarrow \mathbb{C}^{n}, \beta: B^{\prime} \rightarrow \mathbb{C}^{n}$, uniformly close to the identity on their respective domains (depending on $\gamma$ ), and such that

$$
\gamma=\beta \circ \alpha^{-1} \text { on } C^{\prime} .
$$

(This can also be found in Theorem 8.7.2, page 359 in [6].) Choose a simply connected smooth domain $U \subset \mathbb{C}_{n}$ with $\pi_{n}(A) \subset U$ and such that near the origin $U=\left\{z \in \mathbb{C}_{n}: x_{2 n}<0\right\}$. For $j \in \mathbb{N}$ let $l_{j}$ denote the line segment $l_{j}=$ $\left\{z_{n} \in \mathbb{C}_{n}: x_{2 n-1}=0,0 \leq x_{2 n} \leq 1 / j\right\}$. For each $j$ it follows from Mergelyan's Theorem that we may choose injective holomorphic maps $\sigma_{j}: \bar{U} \cup l_{j} \rightarrow \mathbb{C}_{n}$ such that $\sigma_{j}$ approximately stretches $l_{j}$ to cover $\Gamma_{0}$ such that $\sigma_{j}(z)=(1-$ $1 / j) i+z+O(|z-i / j|)^{2 k+1}$ and such that $\sigma_{j} \rightarrow i d$ on $\bar{U}$ as $j \rightarrow \infty$. For each $j$ let $U_{j}$ be a domain obtained from $\mathrm{U}$ by adding a strip around $l_{j}$ of width less than $1 / j$ which is then smoothened and made strictly convex at the end point $l_{j} . U_{j}$ should lie inside where $\sigma_{j}$ is injective holomorphic, and be chosen such that $\sigma_{j}\left(U_{j}\right)$ is strictly convex near the end point of $\sigma_{j}\left(l_{j}\right)=f_{n}$ and such that $\operatorname{Im}\left(\sigma_{j}\left(z_{n}\right)\right)<1$ for all $z \in \overline{U_{j}} \backslash \frac{1}{j} f_{n}$. Let $\psi_{j}$ be a holomorphic diffeomorphism from $\bar{U}$ to $\bar{U}_{j}$ such that $\psi_{j}(0)=\frac{i}{j}, \psi_{j} \rightarrow i d$ uniformly on $\bar{U}$. (See Goluzin, [10], Theorem 2, p. 59.) Let $\phi_{j}=\sigma_{j} \circ \psi_{j}$ and let $\gamma_{j}$ be an extension of $\phi_{j}$ to A. Then $\operatorname{Im}\left(\Pi_{n}\left(\gamma_{j}(z)\right)\right)<1$ for all $z \in A \backslash\{0\}$. It is not hard to see that $\gamma_{j}(A)$ is strictly k-convex near $f_{n}$ and $\gamma_{j} \rightarrow i d$ on a neighborhood of $C$. We get splittings

$$
\gamma_{j} \circ \alpha_{j}=\beta_{j}
$$

as explained above. If $j$ is large enough, we get that (7) defines an injective holomorphic map $f_{j}$ on $\bar{\Omega}$, and if $\alpha_{j}$ is close enough to the identity, since $\alpha_{j}$ can be assumed to vanishes to order $2 k+1$ at the origin, we get that $\operatorname{Im}\left(f_{j}(z)\right)<1$ for all $z \in A \backslash\{0\}$ and such that $f_{j}(A)$ is strictly k-convex at $f_{j}(0)$. 


\section{Andersén-Lempert with parameters in a smooth manifold, and approximation with jet interpolation.}

A parameter version of the Andersen-Lempert theorem [1 for holomorphic parameters was proved by Kutzschebauch[11. Jet interpolation results without parameters have been proved by Forstnerič [9] and Weickert [13] (see also sections 4.9 and 4.15 in [6]). For a smooth manifold $M$ we let $(\zeta, z)$ denote the coordinates on $M \times \mathbb{C}^{n}$. For any $\zeta \in M$ we denote by $\mathbb{C}_{\zeta}^{n}$ the slice $\{\zeta\} \times \mathbb{C}^{n}$, and for any subset $\Sigma \subset M \times \mathbb{C}^{n}$ we let $\Sigma_{\zeta}$ denote the slice $\Sigma_{\zeta}:=\mathbb{C}_{\zeta}^{n} \cap \Sigma$.

Theorem 4.1 Let $M$ be a compact smooth manifold and let $\Omega \subset M \times \mathbb{C}^{n}$ be a domain, $n \geq 2$. Let $K \subset \Omega$ be a compact set, and let $\phi:[0,1] \times \Omega \rightarrow M \times \mathbb{C}^{n}$ be a $\mathcal{C}^{2}$-smooth map such that, writing $\phi(t, \zeta, z)=\phi_{t}(\zeta, z)$, the following hold

(1) $\phi_{t}(\zeta, z)=\left(\zeta, \varphi_{t}(\zeta, z)\right)=\left(\zeta, \phi_{t, \zeta}(z)\right)$

(2) $\phi_{t, \zeta}: \Omega_{\zeta} \rightarrow \mathbb{C}_{\zeta}^{n}$ is injective holomorphic, and

(3) $K_{t, \zeta}:=\phi_{t, \zeta}\left(K_{\zeta}\right)$ varies continuously with $(t, \zeta)$ and is polynomially convex for all $t \in[0,1], \zeta \in M$.

Then $\phi_{1}$ is uniformly approximable on $K$ by a smooth family $\psi(\zeta, z)$ with $\psi_{\zeta} \in$ Aut ${ }_{h o l} \mathbb{C}_{\zeta}^{n}$ if (and only if) $\phi_{0}$ is approximable by such a family. Moreover, if (1)-(3) hold and if $a(\zeta) \in K_{\zeta}^{\circ}$ is a smoothly parametrized family of points, and if $d \in \mathbb{N}$, we may additionally achieve that

(4) $\phi_{1, \zeta}(z)-\psi_{\zeta}(z)=O\left(\|z-a(\zeta)\|^{d}\right)$, as $z \rightarrow a(\zeta)$.

Proof: We give a sketch of the proof of the first claim; the point is just to verify that the non-parametric proof goes through without change with parameters.

The assumption that $\phi_{0}$ is approximable allows us to assume $\phi_{0}=i d$. Define first a parametrized vector field

$$
X_{t, \zeta}\left(\phi_{t, \zeta}(z)\right):=\frac{d}{d t} \phi_{t, \zeta}(z)
$$

Then $X_{t, \zeta}$ is an inhomogeneous vector field, holomorphic in $z$, whose flow is $\phi_{t, \zeta}(z)$. For each $t$ let $\varphi_{t, \zeta}^{s}$ denote the time-s flow of the homogenous vector field $X_{t, \zeta}$ where $t$ is fixed. It is well known that there is a partitioning $[j / n,(j+$ $1) / n], j=0, \ldots, n-1$ of $[0,1]$, such that the composition

$$
\varphi_{(n-1 / n, \zeta)}^{1 / n} \circ \cdots \circ \varphi_{0, \zeta}^{1 / n}
$$

approximates $\phi_{\zeta, 1}$ on $K$. So the problem is reduced to approximating the flow $\varphi_{\zeta}^{1}$ of a homogenous vector field $X_{\zeta}$ on a family $K_{\zeta}$.

Next, by assumption (3) and approximation, we may assume that $X_{\zeta}$ is a polynomial vector field

$$
X_{\zeta}(z)=\sum_{j=1}^{N} g_{j}(\zeta) X_{j}(z),
$$


with coefficients $g_{j}$ in $\mathcal{E}(M)$; this can be obtained by gluing a fiberwise Rungeapproximation using a partition of unity on $M$. Now the main point of AndersénLempert Theory in $\mathbb{C}^{n}$ is that any $m$-homogenous polynomial vector field $V_{m}$ is a sum of completely integrable vector fields (see e.g. [6], Lemma 4.9.5):

$$
V_{m}(z)=\sum_{i=1}^{r} c_{i} \lambda_{i}(z)^{m} \cdot v_{i}+d_{i} \lambda_{i}(z)^{m-1}\left\langle z, v_{i}\right\rangle \cdot v_{i}
$$

with $c_{i}, d_{i} \in \mathbb{C}, v_{i} \in \mathbb{C}^{n}$ and $\lambda_{i} \in\left(\mathbb{C}^{n}\right)^{*}$ with $\lambda_{i}\left(v_{i}\right)=0$. The flows of these two types of vector fields are

$$
z \stackrel{f_{t, j}}{\mapsto} z+t \cdot c_{i} \lambda_{i}(z)^{m} \cdot v_{i} \text { and } z \stackrel{g_{t, j}}{\mapsto} z+\left(e^{t d_{i} \lambda_{i}(z)^{m}}-1\right)\left\langle z, v_{i}\right\rangle \cdot v_{i} .
$$

Applying this to each of the vector fields $X_{j}(z)$ in (9) we get that

$$
X_{\zeta}(z)=\sum_{j=1}^{\tilde{N}} \tilde{g}_{j}(\zeta) \cdot \tilde{X}_{j}(z)
$$

where each $\tilde{X}_{j}$ is completely integrable with flow $\psi_{j}^{s}$, and so $X_{\zeta}$ is a sum of completely integrable fields with flows $\psi_{\zeta, j}^{s}=\psi_{j}^{g(\zeta) \cdot s}$. Finally the sequence

$$
\left(\psi_{\zeta, N}^{1 / n} \circ \cdots \circ \psi_{\zeta, 1}^{1 / n}\right)^{n}
$$

converges uniformly to $\varphi_{\zeta}^{1}$ as $n \rightarrow \infty$.

Finally we consider (4). We will correct the initial approximation at $a(\zeta)$ and by translation we may assume that $a(\zeta)=0$ for all $\zeta$, and that both $\phi$ and $\psi$ fix the origin. Define $J_{d-1}(\zeta)$ to be the $(d-1)$-jet of $\psi_{\zeta}^{-1} \circ \phi_{1, \zeta}$. It is easy to see that we may assume that $J_{d-1}(z)=i d+$ h.o.t, and by the Cauchy estimates we may assume that $J_{d-1}(\zeta)$ is arbitrarily close to the identity map. We will correct $\psi_{\zeta}$ inductively, and our induction assumption is that $J_{d-1}(\zeta)=O\left(\|z\|^{m}\right), 2 \leq m \leq d-1$.

Using (10) we fix an expansion

$$
z^{\alpha} \cdot e_{j}=s_{\alpha, j}(z):=\sum_{i=1}^{r} c_{i}^{\alpha, j} \lambda_{i}^{\alpha, j}(z)^{m} \cdot v_{i}^{\alpha, j}+d_{i}^{\alpha, j} \lambda_{i}^{\alpha, j}(z)^{m-1}\left\langle z, v_{i}^{\alpha, j}\right\rangle \cdot v_{i}
$$

for each multi-index $|\alpha|=m$ and $j=1, \ldots, n$. Now expand the $m$-homogenous part $J_{d-1, m}$ of $J_{d-1}$ using (14)

$$
J_{d-1, m}(\zeta)=\sum_{|\alpha|=m, 1 \leq j \leq n} h_{\alpha, j}(\zeta) \cdot s_{\alpha, j}(z)
$$

It is easy to see that the composition $\Phi_{m}$ of all automorphisms

$$
z \mapsto z+h_{\alpha, j}(\zeta) \cdot c_{i}^{\alpha, j} \lambda_{i}^{\alpha, j}(z)^{m} \cdot v_{i}^{\alpha, j}
$$


and

$$
z \mapsto z+\left(e^{h_{\alpha, j}(\zeta) d_{i}^{\alpha, j} \lambda_{i}^{\alpha, j}(z)^{m}}-1\right)\left\langle z, v_{i}^{\alpha, j}\right\rangle \cdot v_{i}^{\alpha, j} .
$$

matches $J_{d-1, m}$ to order $m$, and we may assume that $\Phi_{m}$ is as close to the identity as we like on a compact set since all the $h_{\alpha, j}$ 's can be assumed to be as small as we like. It follows that the map $\psi_{\zeta} \circ \Phi_{m}$ is a small perturbation of $\psi_{\zeta}$ which matches $\phi_{1, \zeta}$ to order $m$. The induction step is complete.

Remark 4.2 For a more detailed explanation of jet-completion (without parameters) the reader can consult [6] page 154-158.

\section{The construction with parameters: Proof of Theorem 1.4}

Theorem 5.1 Let $\Omega \subset \mathbb{C}^{n}$ be a smooth and bounded convex domain of finite type $2 k$. There exists a smooth parameter family $\psi_{\zeta} \in A u t_{h o l} \mathbb{C}^{n}, \zeta \in b \Omega$, such that $\psi_{\zeta}(\zeta)$ is a globally exposed $2 k$-convex boundary point for the domain $\psi_{\zeta}(\Omega)$.

Proof: By [4] there exist $r, c>0$ and a smooth parameter family

$$
\psi_{\zeta}(z) \text { defined on }\{(\zeta, z):\|z-\zeta\|<r\}
$$

such that $\psi(\zeta, \cdot)$ is injective holomorphic for all $\zeta$ and the following holds for all $\zeta$ (see Section 2): let $n_{\zeta}$ denote the outward pointing unit normal vector to $b \Omega$ at $\zeta$, let $l_{\zeta}$ be a composition of a translation and a unitary transformation such that $l_{\zeta}(\zeta)=0$ and such that $n_{\zeta}$ is sent to the vector $(1,0, \ldots, 0)$. Then $\tilde{\psi}_{\zeta}(z):=\psi_{\zeta} \circ l_{\zeta}^{-1}$ is of the form $\left(S_{\zeta}(z), z_{2}, \ldots, z_{n}\right)$, and $S$ satisfies

$$
S_{\zeta}(z)=3 z_{1}+K z_{1}^{2}+g_{\zeta}\left(z^{\prime}\right), z=\left(z_{1}, z^{\prime}\right),
$$

(See Section 2.) Moreover, we have that

$$
\operatorname{Re}\left(S_{\zeta}(z)\right) \leq-c \cdot\left(\left|z_{1}\right|^{2}+\left\|z^{\prime}\right\|^{2 k}\right), z \in B_{r}(\zeta) \cap \bar{\Omega},
$$

where the constant $c>0$ does not depend on $\zeta$.

Our first step is to change the maps $\psi_{\zeta}$ conveniently on the normals $n_{\zeta}$, and then approximate the changed maps by a family of holomorphic automorphisms. Set $\Gamma_{0}:=\left\{z \in \mathbb{C}^{n}: 0 \leq x_{1} \leq 1, x_{2}=z_{2}=\ldots=z_{n}=0\right\}$, and let $h$ denote the map $h(z)=3 z_{1}+K z_{1}^{2}$ near $\Gamma_{0}$. By changing $h$ smoothly, then finding a smooth homotopy of maps, and finally applying Mergelyan's Theorem with parameters, we find $\delta>0$ and a smooth map

$$
\tilde{h}:[0,1] \times \Gamma_{0}(\delta) \rightarrow \mathbb{C},
$$

such that the following hold

(1) $\tilde{h}_{0}\left(z_{1}\right)=3 z_{1}$,

(2) $\tilde{h}_{t}(\cdot)$ is injective holomorphic for each $t \in[0,1]$, 
(3) $\tilde{h}_{1}\left(z_{1}\right) \approx h\left(z_{1}\right)$ on $B_{\delta}(0)$ and $\left(\tilde{h}_{1}-h\right)(z)=O\left(|z|^{2 k+1}\right)$ as $z \rightarrow 0$,

(4) $\tilde{h}_{1}(z) \approx 3 z_{1}$ on $B_{\delta}(1)$ and $\left(\tilde{h}_{1}-3\right)(z)=O\left(|z-1|^{2 k+1}\right)$ as $z \rightarrow 1$,

(5) $\tilde{h}_{1}^{-1}\left(3 \Gamma_{0}\right) \approx \Gamma_{0}$.

We define a homotopy modification $\widehat{\psi}_{\zeta, t}(z)$ of $\psi_{\zeta}$ by setting

$$
\widehat{S}_{\zeta, t}(z):=\tilde{h}_{t}\left(z_{1}\right)+t \cdot g_{\zeta}\left(z^{\prime}\right) \text { on } \Gamma_{0}(\delta) .
$$

in local coordinates.

Let $b(\zeta)$ denote the end point of $n_{\zeta}$ other than $\zeta$, and note that by Stolzenberg 12 we may assume, by possibly having to decrease $\delta$, that

$$
\widehat{K}_{\zeta, t}:=\widehat{\psi}_{\zeta, t}\left(\overline{B_{\delta}(\zeta) \cup n_{\zeta} \cup B_{\delta}(b(\zeta)}\right)
$$

is polynomially convex for all $\zeta$. By Theorem 4.1 and its proof there exist families $G_{\zeta}, H_{\zeta} \in A u t_{h o l} \mathbb{C}^{n}$ such that the following holds

(6) $G_{\zeta} \approx \widehat{\psi}_{\zeta, 1}$ on $B_{\delta}(\zeta)$, and $\left(G_{\zeta}-\widehat{\psi}_{\zeta}\right)(z)=O\left(\|z-\zeta\|^{2 k+1}\right)$ as $z \rightarrow \zeta$,

(7) $H_{\zeta} \approx \widehat{\psi}_{\zeta, 1}^{-1}$ on $B_{\delta}(0) \cup 3 n_{\zeta} \cup B_{\delta}(3 b(\zeta))$,

(8) $\left(H_{\zeta}-\widehat{\psi}_{\zeta, 1}^{-1}\right)(z)=O\left(\|z-3 b(\zeta)\|^{2 k+1}\right)$ as $z \rightarrow 3 b(\zeta)$, and

(9) $H_{\zeta} \circ G_{\zeta} \approx i d$ on $\bar{\Omega}$.

Next we construct a continuous parameter family of exposing maps $f_{\zeta}$ as in Lemma 3.3, where each $f_{\zeta}$ wraps the boundary at $G_{\zeta}(\zeta)$ around the normal $3 n_{\zeta}$. The composition $H_{\zeta} \circ f_{\zeta} \circ G_{\zeta}$ will globally expose the point $\zeta 2 k$-convexly. We will then change $f_{\zeta}$ to depend smoothly on $\zeta$, and in a final step we will approximate the family $f_{\zeta}$ by a smooth family of automorphisms.

Choose a strictly pseudoconvex neighborhood $\Omega^{\prime}$ of $\bar{\Omega}$ close to $\Omega$ and let $\rho$ be a smooth strictly plurisubharmonic defining function for $\Omega^{\prime}$ near $b \Omega^{\prime}$. For $0<r<<1$ we let $\Omega^{\prime}(r):=\{z: \rho(z)<r\}$. For $0<\sigma<<1$ we define Cartan pairs $\tilde{A}_{\zeta}(r):=\Omega^{\prime}(r) \cap \bar{B}_{\sigma}(\zeta), \tilde{B}_{\zeta}(r):=\Omega^{\prime}(r) \backslash B_{\sigma / 2}(\zeta)$. Set $A_{\zeta}(r):=$ $G_{\zeta}\left(\tilde{A}_{\zeta}(r)\right), B_{\zeta}(r):=G_{\zeta}\left(\tilde{B}_{\zeta}(r)\right)$.

Let $\gamma_{j}$ be the sequence of locally exposing maps from the proof of Lemma 3.3 , Since the maps only depend on the normal coordinate, the map $\tilde{\gamma}_{j, \zeta}:=l_{\zeta}^{-1} \circ \gamma_{j} \circ l_{\zeta}$ is a well defined family of locally exposing maps for $\Omega$, and $\tilde{\gamma}_{j, \zeta} \rightarrow i d$ uniformly on $C_{\zeta}(r):=A_{\zeta}(r) \cap B_{\zeta}(r)$ for small enough $r$ independently of $\zeta$. To globalize these locally defined maps we use the following parametric version of Theorem 8.7.2 in [6].

Lemma 5.2 If $r_{0}$ is small enough and $\mu>0$ there exist $r_{1}<r_{0}$ and $\epsilon>0$ such that the following holds: for any family $\gamma_{\zeta}: \bar{C}_{\zeta}\left(r_{0}\right) \rightarrow \mathbb{C}^{n}$ of holomorphic maps with $\left\|\gamma_{\zeta}-i d\right\|_{\bar{C}_{\zeta}\left(r_{0}\right)}<\epsilon$, continuous in $\zeta$, there exist injective holomorphic maps $\alpha_{\zeta}: A_{\zeta}\left(r_{1}\right) \rightarrow \mathbb{C}^{n}, \beta_{\zeta}: B_{\zeta}\left(r_{1}\right) \rightarrow \mathbb{C}^{n}$, continuous in $\zeta$, such that

$$
\gamma_{\zeta}=\beta_{\zeta} \circ \alpha_{\zeta}^{-1},\left\|\alpha_{\zeta}-i d\right\|_{A_{\zeta}\left(r_{1}\right)}<\mu,\left\|\beta_{\zeta}-i d\right\|_{B_{\zeta}\left(r_{1}\right)}<\mu .
$$

Moreover, we may achieve that $\left(\alpha_{\zeta}-i d\right)(z)=O\left(\|z-\zeta\|^{2 k+1}\right)$ as $z \rightarrow \zeta$. 
The proof of this is almost identical to that in [6], noting that there exist a solution operator to the $\bar{\partial}$-equation which is continuous with parameters, and one can multiply by powers of $S_{\zeta}$ to get exact jet interpolation.

So if $j$ is chosen large enough we get that the family $f_{\zeta}$ defined as $f_{\zeta}:=$ $\gamma_{\zeta, j} \circ \alpha_{j, \zeta}$ on $A_{\zeta}\left(r_{1}\right)$ and $f_{\zeta}:=\gamma_{\zeta, j} \circ \beta_{\zeta, j}$ on $B_{\zeta}\left(r_{1}\right)$, is a family of injective holomorphic maps $\tilde{\gamma}_{\zeta}: G_{\zeta}\left(\Omega^{\prime}\left(r_{1}\right)\right) \rightarrow \mathbb{C}^{n}$ exposing the point $\zeta 2 k$-convexly. By (5) and (8) the family $H_{\zeta} \circ f_{\zeta} \circ G_{\zeta}$ is a continuous family of holomorphic injections on $\Omega^{\prime}\left(r_{1}\right)$, globally exposing the point $\zeta$ for the domain $\Omega$.

Next we approximate $f_{\zeta}$ by a smooth family of exposing maps. This is done using a partition of unity on $b \Omega$. Note first that although $f_{\zeta}$ is only continuous in $\zeta$, the $2 k$-jet at $\zeta, J(\zeta)$, is smooth in $\zeta$; this is because $\alpha_{j, \zeta}$ vanishes to order $2 k$ at $\zeta$. Let $\left(U_{j}, \alpha_{j}\right), j=1, \ldots, m$, be a partition of unity on $b \Omega$ with a point $a_{j} \in U_{j}$ for all $j$. For each $j$ write $f_{a_{j}}(z)=z+g_{j}(z)$. We set $\tilde{f}_{\zeta}(z):=z+\sum_{j=1}^{m} \alpha_{j}(\zeta) g_{j}(z)$. By choosing the covering fine enough we may achieve that $\tilde{f}_{\zeta}$ is as close to $f_{\zeta}$ as we like on $\bar{\Omega}$, and also that the $2 k$-jet of $\tilde{f}_{\zeta}$ at $\zeta$ is as close to that of $f_{\zeta}$ as we like. So using the argument in the proof of Theorem 4.1 we can correct $\tilde{f}_{\zeta}$ so that its $2 k$-jet at $\zeta$ matches that of $f_{\zeta}$ exactly.

Finally we need to approximate the family $\tilde{f}_{\zeta}$ by a family of automorphisms. We may assume that $0 \in \Omega, G_{\zeta}(0)=0$, and that $f_{\zeta}(0)=0$ for all $\zeta$. Set

$$
\varphi_{t}(\zeta, z):=G_{\zeta}\left(\frac{1}{t} \tilde{f}_{\zeta}\left(t \cdot G_{\zeta}^{-1}(z)\right)\right) .
$$

We may assume that $\tilde{f}_{\zeta}\left(G_{\zeta}(\bar{\Omega})\right)$ is polynomially convex for all $\zeta \in b \Omega$. In that case it follows that there exists some $s>1$ such that $\varphi_{t, \zeta}\left(G_{\zeta}(s \bar{\Omega})\right)$ is polynomially convex for all $t, \zeta$, and so approximation follows by Theorem 4.1 . It is enough to show that $f_{\zeta}\left(G_{\zeta}(\bar{\Omega})\right)$ is polynomially convex.

Fix $\zeta \in b \Omega$. By Stolzenberg [12] we have that $G_{\zeta}(\bar{\Omega}) \cup 3 n_{\zeta}$ is polynomially convex. Let $W_{\zeta}$ be a Runge neighborhood of $K_{\zeta}:=G_{\zeta}(\bar{\Omega}) \cup 3 n_{\zeta}$, very close to $K_{\zeta}$. Consider a point $b \in b \Omega \cap \bar{B}_{\zeta}(0)$. If $W_{\zeta}$ is close enough to $K_{\zeta}$, and if $\beta_{\zeta}$ is close enough to the identity, then the locally defined function $e^{C \cdot S_{b}\left(\beta_{\zeta}^{-1}(z)\right)}$ for $C>>0$ may be approximately globalized to $W_{\zeta}$, separating points on $\beta_{\zeta}\left(n_{b}\right)$ close to $\beta_{\zeta}(b)$ from $f_{\zeta}\left(G_{\zeta}(\bar{\Omega})\right)$ as long as $f_{\zeta}$ is chosen such that $f_{\zeta}(\bar{\Omega}) \subset W_{\zeta}$. It follows that

$$
\left.c l\left[f_{\zeta} \widehat{\left(G_{\zeta}(\bar{\Omega})\right.}\right) \backslash f_{\zeta}\left(G_{\zeta}(\bar{\Omega})\right)\right] \cap f_{\zeta}\left(G_{\zeta}(\bar{\Omega})\right) \subset f_{\zeta}\left(A_{\zeta}(0)\right) .
$$

Hence by Rossi's local maximum principle

$$
\left.f_{\zeta}\left(\widehat{G_{\zeta}(\bar{\Omega}}\right)\right)=f_{\zeta}\left(G_{\zeta}(\bar{\Omega})\right) \cup\left[f_{\zeta}\left(A_{\zeta} \widehat{(0)) \cap} G_{\zeta}(\bar{\Omega})\right] .\right.
$$

But $f_{\zeta}^{-1}$ is approximable by entire maps on $f_{\zeta}\left(A_{\zeta}(0)\right)$, and so $f_{\zeta}\left(G_{\zeta}(\bar{\Omega})\right)$ is polynomially convex.

\section{Remark on the proof of Theorem 1.3}

The proof of Theorem 1.3 is almost the same as that of Theorem 1.1. except that we need to make sure that the exposing maps $f_{j}$ are approximable by 
holomorphic automorphisms. To see why this is so, note first that each $\gamma_{j}$ may be connected to the identity map by an isotopy which is uniformly close to the identity on $C$. The Cartan type splitting with parameters then allows us to construct each $f_{j}$ as the time- 1 map of an isotopy $f_{j, t}$ with $f_{j, 0}=$ id (this argument allows us to avoid the usual assumption in Andersén-Lempert theory that $\Omega$ is star shaped). This isotopy is only $\mathcal{C}^{0}$ but we can obtain a smooth isotopy by gluing as before. The same argument as in the previous section tells us that we may assume that $f_{t, j}(\bar{\Omega})$ is polynomially convex for all $t$ if $j$ is sufficiently large, and so we may approximate by automorphisms.

Acknowledgement 6.1 The authors would like to thank the referee for a very carefull reading, thus helping us to improve greatly the exposition of the paper.

\section{References}

[1] E. Andersén and L. Lempert, On the group of holomorphic automorphisms of $\mathbb{C}^{n}$, Invent. Math. 110 (1992), 371-388.

[2] D'Angelo, John P: Several complex variables and the geometry of real hypersurfaces. Studies in Advanced Mathematics. CRC Press, Boca Raton, FL, 1993.

[3] F. Deng, Q. Guan, and L. Zhang, On some properties of squeezing functions on bounded domains, Pac. J. Math. 257 (2012), 319-341.

[4] K. Diederich and J.E. Fornæss, Support functions for convex domains of finite type, Math. Z. 230 (1999), 145-164.

[5] F. Forstnerič, Noncritical holomorphic functions on Stein manifolds, Acta Math. 191 (2003), 143-189.

[6] F. Forstnerič, Stein Manifolds and Holomorphic Mappings, Ergebnisse der Mathematik und ihrer Grenzgebiete, 3. folge 56. Springer-Verlag, BerlinHeidelberg 2011.

[7] F. Forstnerič and E. Løw, Global holomorphic equivalence of smooth manifolds in $\mathbb{C}^{k}$, Indiana University Math. J. 46 (1997), 133-153.

[8] F. Forstnerič and E. F. Wold, Bordered Riemann Surfaces in $\mathbb{C}^{2}$, J. Math Pures Appl. 91 (2009.1), 100-114.

[9] F. Forstnerič, Interpolation by holomorphic automorphisms and embeddings in $\mathbb{C}^{n}$. J. Geom. Anal. 9 (1999), no. 1, 93-117.

[10] G. Goluzin, Geometric theory of functions of a complex variable, Amer. Math, Soc., Providence, R.I., 1969.

[11] F. Kutzschebauch, Andersen-Lempert Theory with Parameters: A Representation Theoretic Point of View, J. Alg. Appl. 4 (2005), 325-340. 
[12] G. Stolzenberg, Uniform approximation on smooth curves, Acta Math. 115 (1966), 185-198.

[13] Weickert, B.; Ph. d. Thesis, University of Michigan (1997). 